\title{
Study on applicability of two modal identification techniques in irrelevant cases
}

\author{
A. Tomaszewska' ${ }^{1} \cdot$ M. Szafrański² ${ }^{2}$
}

Received: 22 January 2020 / Revised: 22 January 2020 / Accepted: 1 February 2020 / Published online: 17 February 2020

(c) The Author(s) 2020

\begin{abstract}
Study on applicability of two modal identification techniques in irrelevant cases is made in this paper. The following techniques are considered: peak picking based on correlation analysis (PP-CA), dedicated for ambient vibrations and eigensystem realization algorithm (ERA), formulated for free-decay vibrations investigation. Irrelevant cases are found when analyzed signals are different than recommended to a given technique. The study is conducted on examples of two real structures: masonry tower and steel railway bridge. Both cases are diverse in age, material, excitation and vibrations energy. The signals measured on the tower are suitable for the PP-CA technique (ambient vibrations), while the signals measured on the bridge are suitable for the ERA (free-decay vibrations). However, both methods have been applied to both systems. Natural frequencies, mode shapes and damping ratios are identified and the effectiveness of the irrelevant technique is assessed in relation to the results obtained by the relevant method in each case.
\end{abstract}

Keywords Modal identification $\cdot$ Structural dynamics $\cdot$ Ambient vibrations $\cdot$ Free-decay vibrations $\cdot$ Engineering structures

\section{Introduction}

The methodology of modal parameters identification of existing structures consists of three general steps: (a) measurements performance-data collection, (b) signal processing - applying of selected modal identification method (MID) for modal parameters extraction, and (c) verification of the results. Extensive classification of MID techniques due to analysis domain (time or frequency), type and number of inputs and outputs can be found in [1]. MID techniques are a subject of discussion during specialized conferences, see, e.g., [2]. The choice of an appropriate MID technique is affected by the type of applied excitation and the type of

A. Tomaszewska

atomas@pg.edu.pl

M. Szafrański

mszafran@pg.edu.pl

1 Department of Structural Mechanics, Faculty of Civil and Environmental Engineering, Gdańsk University of Technology, Narutowicza 11/12, 80-233 Gdańsk, Poland

2 Department of Bridge Structures and Railway Engineering, Faculty of Civil and Environmental Engineering, Gdańsk University of Technology, Narutowicza 11/12, 80-233 Gdańsk, Poland measured response. Due to structural nonlinearity, environmental impacts and equipment used, the measured signals are always corrupted by noise. The MID methods are sensitive to signal distortions, frequently leading to result discrepancy. The limitation knowledge of a given method helps to distinguish valid and invalid modal identification results. Therefore, in this paper two extreme cases are considered, aimed to discuss the applicability of eigensystem realization algorithm (ERA) and peak picking based on correlation analysis (PP-CA) techniques in irrelevant cases, i.e., to a data that the method is not expected to be performant. The ERA is classified as the multi-input/multi-output experimental modal analysis method in time domain. The algorithm uses free-decay response data occurring after impulse excitation, initial condition or after an immediate interruption of running excitation of any kind (tugging, jumping, hitting, etc.). In this technique, individual signal samples collected by each measuring sensor are selected to form a Hankel matrix. Further matrix operations allow to identify natural frequencies, mode shapes and damping coefficients. The PP is classified as the multi-output operational modal analysis method in frequency domain. The PP-CA is aimed at ambient responses considerations. The measured time series are transformed into frequency domain by means of spectral functions (auto- and cross-spectra). Correlation analysis of 
these functions performed at various structural points allows to identify natural frequencies and corresponding mode shapes. Details of the method are presented in [3]. In the prior description, both selected methods incorporate output structural information only. This is practically convenient while no input information is necessary; so no dedicated exciters with force measuring instrumentation are required, as discussed, e.g., in the paper [4] in a bridge case or in [5] in a historical masonry belfry case. Moreover, the outputonly methods cover large structures like bridges, buildings and towers, whose excitations are difficult to control. In the paper [6], the application of PP-CA method for modal identification of a 141-m-high building is reported. Six modes have been identified. In the Ref. [7], the PP method is used for modal identification of the multi-span concrete Nelson St. bridge in Auckland (New Zealand). Resonant frequencies are identified using the averaged normalized power spectral density function (ANPSD) calculated for the measured ambient responses of the bridge deck. Spectral analysis is also applied to the measurement results of Rędziński Bridge (Poland) [8]. In this case, free response signals collected after the passages of vehicles are considered as an input. The example of the ERA application is presented in, e.g., [9] in the case of suspension New Carquinez Bridge in San Francisco (USA). Based on the ambient vibration measurements, the correlation analysis is applied first to transform the random data into deterministic form-free-decay signals (correlation functions). Then, the ERA technique is used for modal parameters identification. In the Ref. [10] in contrast, the ERA is applied directly to the free-vibration signals of the Radunia River Bridge in Gdansk (Poland) collected after the passages of trains. In the Ref. [11], the output-only data are used directly for modal parameters identification and model updating of the truss railway bridge over Vistula River in Tczew (Poland). Free vibrations caused by trains running on the structure as well as ambient vibrations are taken as the inputs.

In the present study, similar types of responses (free and ambient) are considered as the inputs for ERA and PP-CA techniques are diverse. Short-time signals of decay nature are suitable for the ERA, while the PP-CA requires long time series of ambient vibrations. The advantage of ERA is damping coefficient identification, besides natural frequencies and modes, which are the output of the PP-CA method too. On the other hand, the natural asset of PP-CA is a possibility of statistical error determination of the identified modal parameters. Knowledge of the modal parameters scatters is an important issue, e.g., in structural damage detection. There are attempts to build a technique of damage identification based on scattered modal data. In example, in the paper [12], the authors propose an optimization problem with the objective to minimize a difference between elements of the experimental flexibility matrix and corresponding ones predicted in numerical model. Numerical example shows efficacy of the algorithm, also in a case of scattered modal forms. Similar approach is proposed in earlier paper [13], where the efficacy of classical flexibility and curvature approaches of damage detection but in a condition of scattered modal forms is studied in numerical examples and in a case of masonry tower.

The mentioned different requirements and features of PP-CA and ERA methods are decisive for an individual choice. However, due to given reasons, the MID technique may be selected irrelevant in the analyzed case. In this paper, the consequences of such a choice are discussed in two examples. The real structures are selected to embed the analysis in practical engineering domain. The analysis is naturally affected by noise and uncertainties concerning material and structural integrity. The selected structures differ in construction and environmental influences. The first case is a tower in the Vistula Mounting Fortress in Gdansk, incorporating measurement of ambient vibrations supplemented by short-time interruptions. The second case is a steel railway bridge; here, free-decay vibrations have been measured just after the passage of train. The first example employs the PP-CA technique while the second is related to ERA. Both techniques are applied due to both examples to assess the methods applicability in the irrelevant cases. In each case the results of the properly selected MID technique is a reference to another. Each case includes identification of natural frequencies, mode shapes and damping coefficients.

\section{Materials and methods}

\subsection{Theory of selected modal techniques}

\subsubsection{Eigensystem realization algorithm (ERA)}

The ERA method evolves from the Ho-Kalmann minimum realization problem. It estimates the state-space model:

$$
\begin{aligned}
\mathbf{x}_{k+1} & =\mathbf{A} \mathbf{x}_{k}+\mathbf{B} \mathbf{u}_{k}, \\
\mathbf{y}_{k} & =\mathbf{C} \mathbf{x}_{k}+\mathbf{D} \mathbf{u}_{k},
\end{aligned}
$$

based on finite-time and noisy experimental data [14]. Over the years, the method has been extensively studied and used for modal parameters identification in various engineering problems, e.g., bridges-vehicle dynamic interaction (see, e.g., [10]), high-cycle fatigue of engines (see, e.g., [15]), machines construction optimization (see, e.g., [16]), and damage detection of engineering structures (see, e.g., [17]). The basic principle of ERA is to identify the system matrices $\mathbf{A}, \mathbf{B}$ and $\mathbf{C}$ of order $n$ ( $n$ is the number of significant equations to define the identified system) by means of measured free-vibration response. The algorithm results in 
the minimum realization of a discrete, linear time-invariant system of the $n$-th order defined by the triple:

$$
\begin{aligned}
\mathbf{A}^{e} & =\mathbf{S}_{n}^{-1 / 2} \mathbf{U}_{n}^{T} \mathbf{H}(1) \mathbf{V}_{n} \mathbf{S}_{n}^{-1 / 2}, \\
\mathbf{B}^{e} & =\mathbf{S}_{n}^{1 / 2} \mathbf{V}_{n}^{T} \mathbf{E}_{r}, \\
\mathbf{C}^{e} & =\mathbf{E}_{p}^{T} \mathbf{U}_{n} \mathbf{S}_{n}^{1 / 2},
\end{aligned}
$$

where the matrices $\mathbf{A}^{e}, \mathbf{B}^{e}, \mathbf{C}^{e}$ are estimated quantities, the matrices $\mathbf{S}_{n}, \mathbf{U}_{n}, \mathbf{V}_{n}$ are produced by Singular Value Decomposition of the Hankel matrix $\mathbf{H}(0)$, taking into account $n$ significant results. Auxiliary matrices $\mathbf{E}_{r}$ and $\mathbf{E}_{p}^{T}$ contain an appropriate number of identity and zero matrices of order $p: \mathbf{E}_{r}=\left[\begin{array}{llll}\mathbf{I}_{p} & \mathbf{0}_{p} & \ldots & \mathbf{0}_{p}\end{array}\right]^{\mathrm{T}}, \mathbf{E}_{p}^{\mathrm{T}}=\left[\begin{array}{llll}\mathbf{I}_{p} & \mathbf{0}_{p} & \ldots & \mathbf{0}_{p}\end{array}\right]$, where $p$ is the number of measured signals. An eigenvalue decomposition $\mathbf{A}^{e} \boldsymbol{\Phi}=\boldsymbol{\Phi} \boldsymbol{\Lambda}$ leads to the continuous-time matrix $\boldsymbol{\Lambda}_{c}=f_{s} \ln (\boldsymbol{\Lambda})$ ( $f_{s}$ is the sampling frequency), providing information on eigenfrequencies $f_{n}$ and modal damping $\xi$ of the $i$-th mode:

$\lambda_{c i}, \lambda_{c i}^{*}=-\xi_{i} \omega_{n i} \pm j \omega_{n i} \sqrt{1-\xi_{i}^{2}}$,

where $\omega_{n}$ denotes natural circular frequency and $i=1,2,3, \ldots, p$ ( $p$-number of sensors). The mode shape vectors are linked with the output matrix defined in modal coordinates $\mathbf{C}_{m}^{e}=\mathbf{C}^{e} \boldsymbol{\Phi}$. Each coordinate becomes [18]:

$\varphi_{i j}=\left|c_{m}^{i j}\right| \operatorname{sign}\left\{\operatorname{Re}\left(c_{m}^{i j}\right)\right\}$,

where $j=1,2,3, \ldots, n$. modal amplitude coherence (MAC) of the observability and controllability matrices can be applied to the ERA results to distinguish the true modes from the numerical ones [19]. The MAC criterion checks time compatibility between each individual modal component response of the identified model (vectors $\mathbf{q}_{i}^{\mathrm{e}}$ ) and the real system (vectors $\mathbf{q}_{i}$ ), defined as a normalized scalar product between both vectors:

$\operatorname{MAC}_{i}=\frac{\left|\mathbf{q}_{i} \mathbf{q}_{i}^{e *}\right|}{\sqrt{\left(\left|\mathbf{q}_{i} \mathbf{q}_{i}^{*} \| \mathbf{q}_{i}^{e} \mathbf{q}_{i}^{e *}\right|\right)}}$.

While the MAC value is close to unity, it denotes that both vectors coincide; so the corresponding modal parameters characterize the identified model (they are considered structural). The results of ERA are usually presented graphically on frequency stabilization diagrams. To provide a better result evaluation, the averaged normalized power spectral density (ANPSD) functions are plotted simultaneously for the set of $p$ measured signals:

$\operatorname{ANPSD}\left(f_{k}\right)=\frac{1}{p} \sum_{i=1}^{p} \frac{\operatorname{PSD}_{i}\left(f_{k}\right)}{\sum_{k=1}^{N} \operatorname{PSD}_{i}\left(f_{k}\right)}$, where $f_{k}$ is the $k$-th discrete frequency $(k=1,2, \ldots, N), \mathrm{PSD}_{i}$ is the auto-power spectrum (Power Spectral Density) of the $i$-th signal $(i=1,2, \ldots, p), N$ is the number of discrete frequencies.

\subsubsection{Peak picking based on correlation analysis (PP-CA)}

This method has been introduced in the book [3]. Here, the measured time series are transformed into frequency domain by means of spectral functions. To obtain natural frequencies and mode shapes, the auto- and cross-spectra functions are required due to a given structure, to be computed as follows:

$\hat{G}_{p p}(f)=\frac{2}{n_{d} T} \sum_{k=1}^{n_{d}}\left|P_{k}(f, T)\right|^{2}$,

$\hat{G}_{p q}(f)=\frac{2}{n_{d} T} \sum_{k=1}^{n_{d}} P_{k} *(f, T) Q_{k}(f, T)$,

where $\hat{G}_{p p}(f)$ and $\hat{G}_{p q}(f)$ denote estimate of auto-spectrum and cross-spectrum, respectively, of signals $p(t)$ and $q(t) ; T$ is the measurement time; $n_{d}$ is the number of $p(t)$ and $q(t)$ signals analyzed; $P$ and $Q$ stand for the Fourier transforms of signals $p(t)$ and $q(t)$; denotes the complex conjugate. In practice $n_{d}$ is the number of series the long-time signals are divided into. The true values of spectral functions are computed in an unlimited time domain, which is practically impossible. Thus, the signals measured in limited time are applied to compute the estimates. These estimates are affected by statistical errors. As the autospectra are applied to determine the mode shapes, the errors affect the mode shapes as well. The mode shape coordinates are based on the following formula:

$\hat{\phi}_{p}\left(f_{m}\right)=\sqrt{\frac{\hat{G}_{p p}\left(f_{m}\right)}{\hat{G}_{r r}\left(f_{m}\right)}}$,

where $\hat{\phi}_{p}\left(f_{m}\right)$ is the mode shape coordinate calculated for a structural point $p$ related to the resonant frequency $f_{m}, \hat{G}_{r r}\left(f_{m}\right)$ denotes the auto-spectrum value for $f_{m}$, determined for $r(t)$ signal collected at the structural reference point $r$. The form of the mode shape related to $f_{m}$ (torsional, lateral, etc.) is determined on a basis of the cross-spectra phase shift values in the frequency $f_{m}$. Finally, $f_{m}$ is the frequencies of high (close to unity) values of coherence function $\gamma^{2}(f)$, defined as a quotient of spectral functions, see formula (10).

$\hat{\gamma}_{p q}^{2}(\boldsymbol{f})=\frac{\left|\hat{G}_{p q}(\boldsymbol{f})\right|^{2}}{\hat{G}_{p p}(\boldsymbol{f}) \hat{G}_{q q}(\boldsymbol{f})}, \quad 0 \leq \hat{\gamma}_{p q}^{2}(\boldsymbol{f}) \leq 1$.

The details of the discussed methodology can be found in [3]. 
The statistical error of auto-spectra, required for mode shape identification, is a sum of bias $\varepsilon_{b}$ and random $\varepsilon_{r}$ scatters [20], according to the formula:

$\varepsilon\left[\hat{G}_{p p}(\boldsymbol{f})\right]=\varepsilon_{b}\left[\hat{G}_{p p}(\boldsymbol{f})\right]+\varepsilon_{r}\left[\hat{G}_{p p}(\boldsymbol{f})\right] \approx \frac{\Delta f^{2}}{24}\left[\frac{\left(G_{p p}(\boldsymbol{f})\right)^{\prime \prime}}{G_{p p}(\boldsymbol{f})}\right]+\frac{1}{\sqrt{n_{d}}}$,

where $\Delta f$ means the frequency resolution of the spectra, $\left(G_{p p}(f)\right)^{\prime \prime}$ stands for the second derivative of the $G_{p p}(f)$ function. Finally, the statistical error of mode shape coordinates yields from the relation (12), based on the rules of error transferring, see [21]:

$\varepsilon\left[\hat{\phi}_{p}\right]=\frac{1}{2}\left(\varepsilon\left[\hat{G}_{p p}\right]+\varepsilon\left[\hat{G}_{r r}\right]\right)$.

The statistical error of the identified natural frequencies is composed of two parts: the digitalization error related to the spectrum resolution and the random error related to dispersion of resonant frequencies.

\subsection{Description of selected structures}

\subsubsection{Tower in the Vistula Mounting Fortress in Gdansk}

The tower stands in the center of Vistula Mounting Fortress in Gdansk (Poland), (Fig. 1a). This is a unique structure, one of the most early dated monuments of fortification art in Poland (fifteenth century). At the beginning, the tower was the only structure built at the Vistula river mounting, serving both as a lighthouse and a defensive tower. Throughout the centuries, subsequent buildings have been erected around the tower to improve the object strength. Nowadays, the tower reaches $22.65 \mathrm{~m}$, and its external diameter is $7.7 \mathrm{~m}$. It features six floors of reinforced concrete ceilings. The masonry walls date back to various ages because of war damages and further renovations. The wall thickness is variable, higher near the foundation level and lower at the top. The average thickness is $1.25 \mathrm{~m}$. The tower is founded shallow on weak layered subsoil, see also [22].

\subsubsection{K030 temporary railway bridge span}

The bridge is a temporary simply supported steel railway span (Fig. 1b). It was operated during the construction stage of the new 'Green Route' in Gdańsk (Poland). The route was built under the existing E65 railway line (Warsaw-Gdynia in Poland), the span was located along the line to keep the railway traffic free. The span consists of two steel box girders, 1.86-m wide and $1.28-\mathrm{m}$ high in the mid-span section The girders are fastened transversally by screw joints. The girder cross section varies throughout length due to the use of cover plates. The span is 30-m long and 3.72-m wide. Rails are fastened directly to the structure. The span is supported on concrete abutments by means of steel chock bearings.

\subsection{Dynamic measurements}

\subsubsection{The tower}

Ambient vibrations (accelerations) of the tower are measured with the use of PULSE 3650C system and piezoelectric sensors. Vibrations are caused mostly by wind and paraseismic influences. Special excitations are also applied in the form of a regular hitting of the tower wall at the top floor by a group of people or by a truck driving through an obstacle located next to the tower.

Accelerations measured by sensors located in a single vertical line along the tower height are considered in the study. Vibrations in the direction perpendicular to plane of the selected wall are measured here. Nine sensor locations are set this way. The lowest sensor position is $5.2 \mathrm{~m}$ above the ground level (sensor A1) and the highest is $21.1 \mathrm{~m}$ above the ground (sensor A9), see Fig. 2a. The measurements are made in four series, each series employs three active sensors. The sensor A4, situated $11.5 \mathrm{~m}$ above the ground, is active in all measurements, and considered a reference in all measurements. The sampling frequency is set to $256 \mathrm{~Hz}$. Ambient vibration measurements last $1024 \mathrm{~s}$. They are interrupted by short-time excited vibrations, as stated above. The example signals measured by A9 and A8 sensors are presented in Fig. 3. Wind influence on the sensors is filtered out for
Fig. 1 a Tower in the Vistula Mounting Fortress, b KO30 temporary bridge span

Fig. 2 a The scheme of the tower with the sensors arrangement; $\mathbf{b}$ the first mode shape of the tower identified with the use of PP-CA and ERA techniques (gray area marks statistical error of the estimate by PP-CA technique)

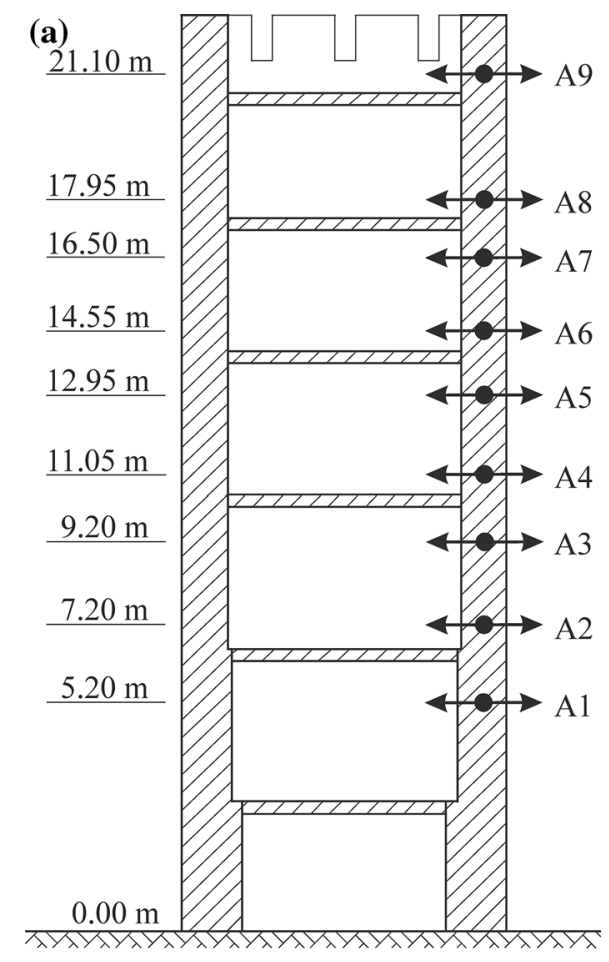

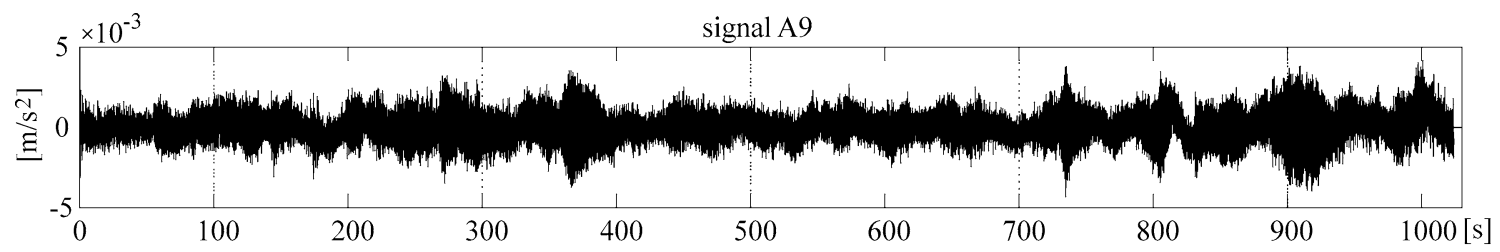

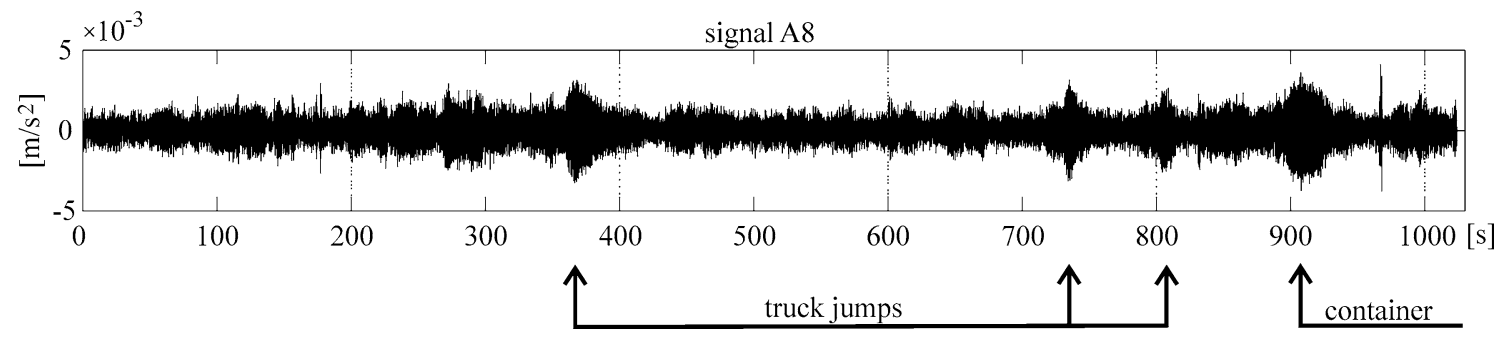

Fig. 3 Example accelerations measured on the tower

this presentation (bandpass $0-0.5 \mathrm{~Hz}$ ). Increased accelerations due to truck jumps or a container flow on the river are featured.

\subsubsection{The bridge}

Vibration measurements of the bridge are performed under a moving ST44 diesel locomotive. This is the six-wheelset locomotive, each wheelset of $194 \mathrm{kN}$ weight. Free response data are collected since the locomotive passes over the bridge with the velocities equal $10,20,30 \mathrm{~km} / \mathrm{h}$ in two directions: to Gdansk and from Gdansk. Vertical accelerations of the mid-span

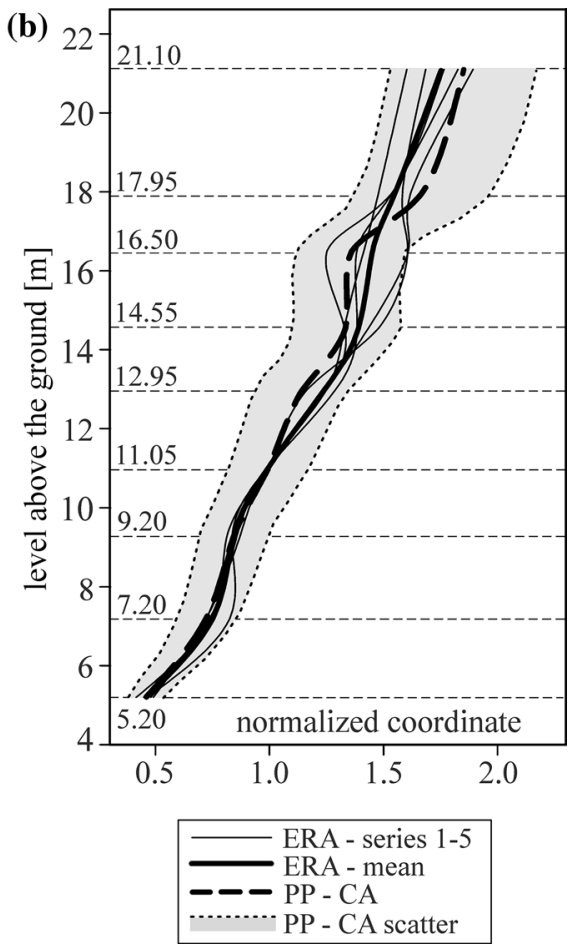




\section{Results}

\subsection{The tower}

The measured signals are applied into ERA and PP-CA algorithms as an input. Both approaches are aimed to identify frequencies and mode shapes. In the ERA, also damping ratios are obtained and compared to the results of logarithmic decrement method, applied separately to parts of signals of decay character. A single natural frequency and a related mode shape are identified due to the tower only. Higher frequencies are barely observable on spectra determined for higher structural levels only. While applying the PP-CA technique, ambient vibration signals are incorporated. They are pre-processed by filtering out frequencies below $0.8 \mathrm{~Hz}$ (wind bandpass). The signals contain $N=262144$ samples. The PP-CA requires splitting the collected signals into $n_{d}$ parts. The selected number is intended to balance the random error of correlation functions $\varepsilon_{r}\left[\hat{G}_{p p}(f)\right]$ and their resolution $\Delta f$-the higher the $n_{d}$ value the lower the error, but while the frequency resolution decreases, the identification accuracy of natural frequencies decreases too. In this case the number $n_{d}=32$ yields a balancing effect: $\varepsilon_{r}\left[\hat{G}_{p p}(f)\right]=1 / \sqrt{32}=17.7 \%$, $\Delta f=256 \cdot n_{d} / N=0.031 \mathrm{~Hz}$. The bias of the mode shape error is around $0.1 \%$, thus negligible. The obtained mode shape and its statistical error are marked in Fig. 2b. The mode is visualized using spline functions to provide interpolation between the measured coordinates.

Due to ERA technique, the measured signals are applied directly, without any filtering. The few-second-long signal intervals of significant amplitude level are selected and processed. These parts of signals are collected after some additional excitation made by a group of people or a truck-see Sect. 2.3. There are five different signal parts, the duration periods are $10 \mathrm{~s}, 7 \mathrm{~s}$ and $4 \mathrm{~s}$; thus, eventually fifteen signals are subjected to the analysis. Each analyzed case results in a frequency and a mode shape. In the case of damping identification, only the 4-s intervals have been analyzed, showing a highly visible decay character (Fig. 4).

The Hankel matrices $(\alpha p \times \beta)$ are built taking into account all selected data: $\alpha=(120 p ; 200 p ; 280 p)$ and $\beta=3 \alpha$, where $p$ is the number of signals included. Three response signals are measured simultaneously; hence, the sizes of Hankel matrices are $[1080 \times 1080 ; 1800 \times 1800 ; 2520 \times 2520]$. A representative frequency stabilization diagram of ERA is shown in Fig. 5a together with ANPSD. Figure 5b shows the comparison in time between the original measured signals and the ERA result (the modal component of the single frequency $f_{1}$ ) obtained for the time interval no. 5 according to Fig. 4 . The mode shapes obtained for the 10 -s signals are presented in Fig. 2b.

The ERA-based natural frequencies cover the range of $1.44-1.46 \mathrm{~Hz}$, their mean value is $1.45 \mathrm{~Hz}$, standard deviation is 0.0113 . The result of PP-CA algorithm is also $1.45 \mathrm{~Hz}$. The accuracy of the PP-CA result is $0.015 \mathrm{~Hz}$, half the $\Delta f$ value. The correlation coefficients of the mode shape vectors calculated for each subsequent result obtained in ERA in relation to the reference PP-CA result belong to the interval (0.9917-0.9969). The damping ratios identified with the use of ERA and logarithmic decrement method are 0.0217 and 0.0241 , respectively. All the results are summarized in Table 1.

\subsection{The bridge}

A single test allows to identify the three natural frequencies and the related mode shapes of the bridge. Measurements are performed after the passage of train with the velocity of $10 \mathrm{~km} / \mathrm{h}$ (see signals in Fig. 6). Considering other velocities, only the first natural frequency and the related mode shape could be identified. The response signals have been processed by ERA and PP-CA techniques; however, their free-decay character and short measurement time suit the ERA algorithm most of all. Similar to previous case, in ERA algorithm, Hankel matrices are built to take into
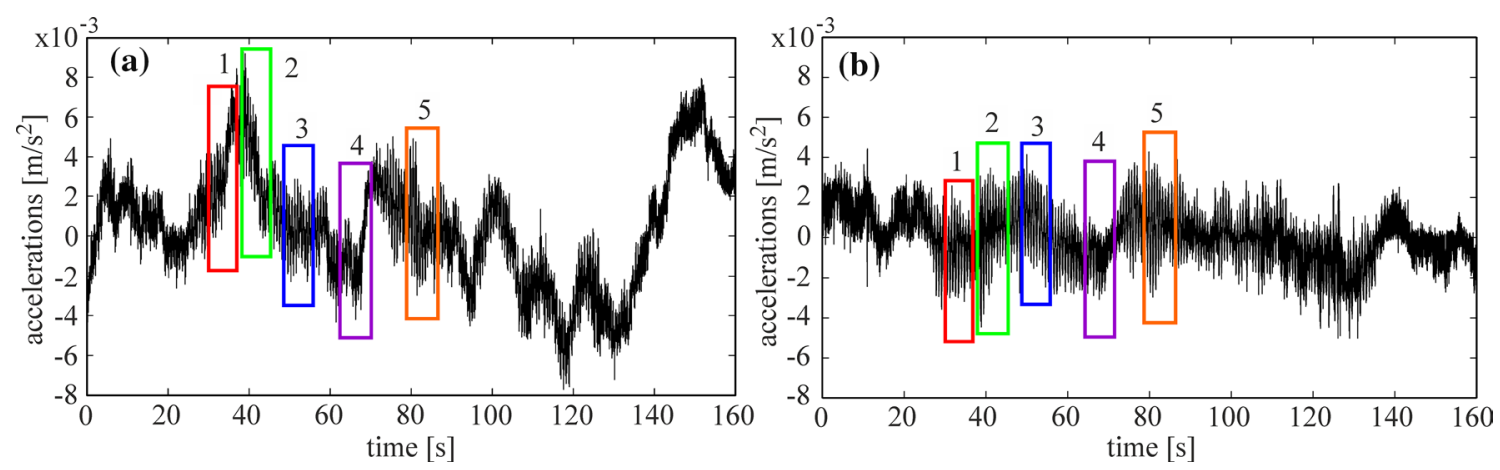

Fig. 4 Response of horizontal acceleration of the tower with selected signal intervals (1-5) for ERA calculations: a sensor A4, b sensor A5 


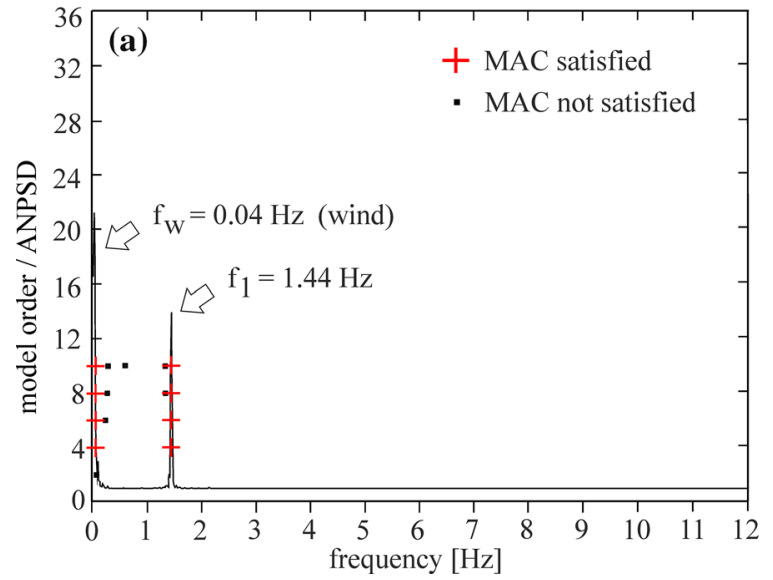

Fig. 5 a Representative frequency stabilization diagram of the tower (ERA) together with ANPSD function-series 1, sensors A9, A8, A4; b free response of the tower in sensors A4 and A5 for the time inter-

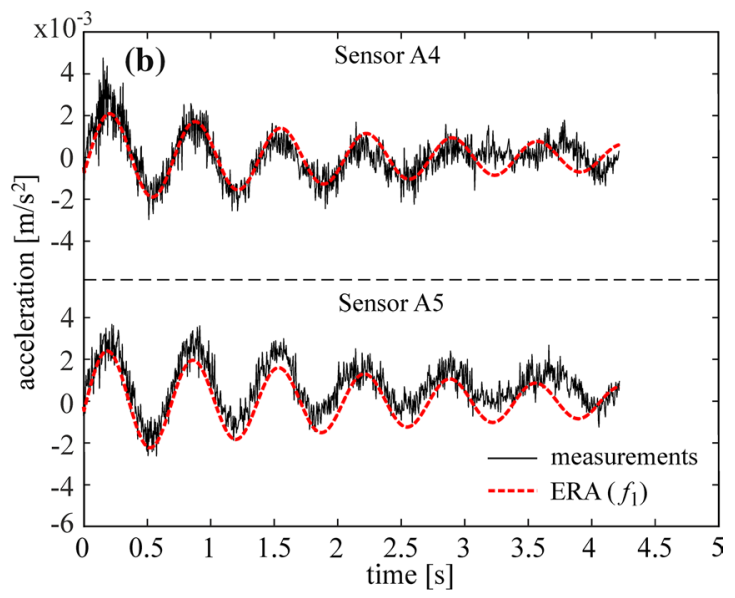

val no. 5 according to Fig. 4-original measurements versus ERA (identified mode of the single frequency $f_{1}$ )
Table 1 Results of modal parameters identification of the considered structures

\begin{tabular}{|c|c|c|c|c|c|c|c|c|}
\hline & \multicolumn{5}{|l|}{ The bridge } & \multicolumn{3}{|l|}{ The tower } \\
\hline & $f_{1}(\mathrm{~Hz})$ & $f_{2}(\mathrm{~Hz})$ & $f_{3}(\mathrm{~Hz})$ & $\xi_{1}(-)$ & $\xi_{1}^{\mathrm{LDD}}(-)$ & $f_{l}(\mathrm{~Hz})$ & $\xi_{1}(-)$ & $\xi_{1}^{\mathrm{LDD}}(-)$ \\
\hline ERA & 3.98 & 13.29 & 25.48 & 0.0059 & 0.0061 & 1.45 & 0.0217 & 0.0241 \\
\hline PP-CA & $4.26 \pm 0.35$ & $13.49 \pm 0.35$ & $25.57 \pm 0.35$ & N.A. & & $1.45 \pm 0.015$ & N.A. & \\
\hline
\end{tabular}



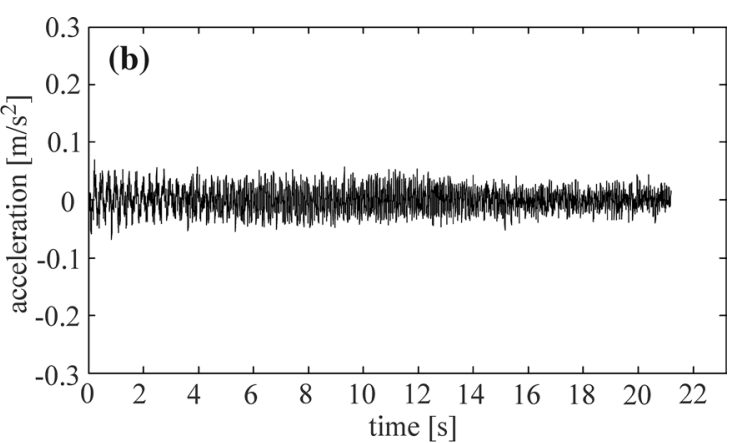

Fig. 6 Free response of vertical acceleration of the bridge after the passage of the locomotive with the velocity of $10 \mathrm{~km} / \mathrm{h}$ : a mid-span section, $\mathbf{b}$ quarter-span section

account significant response level (signals of an approximate 4-s length have been chosen). Four response signals are applied into the algorithm, so the size of the Hankel matrix is $960 \times 720$ according to $\alpha=60 p, \beta=3 \alpha$. The frequency stabilization diagram of ERA with ANPSD function is plotted in Fig. 7. The mode shapes are presented in Fig. 8b, considering the entire, symmetric bridge span. The spline functions are applied to interpolate between the identified coordinates.

In the case of the PP-CA technique the same signals are considered, however their entire length is applied to the analysis. The signals last $21.192 \mathrm{~s}$; the number of samples is $N=5298$ samples. The signals are divided into $n_{d}=15$ parts; this is a compromise between random error of mode shape coordinates $\left(\varepsilon_{r}\left[\hat{G}_{p p}(f)\right]=1 / \sqrt{15}=25.8 \%\right)$ and the spectral resolution $\Delta f=0.71 \mathrm{~Hz}$. Such a set-up makes the obtained mode shapes resemble the ERA-based modes closely. The correlation coefficient between the results of both algorithms is $0.9999,0.9929,0.9987$ for the subsequent modes. The mode shapes identified in PP-CA are presented in Fig. 6b as well. The results of ERA and PP-CA are similar; so the statistical error determined in the PP-CA algorithm is not marked in the figure.

The natural frequencies identified by both methods are also similar-the values indicated by ERA are: $f_{1}=3.98 \mathrm{~Hz}$, 


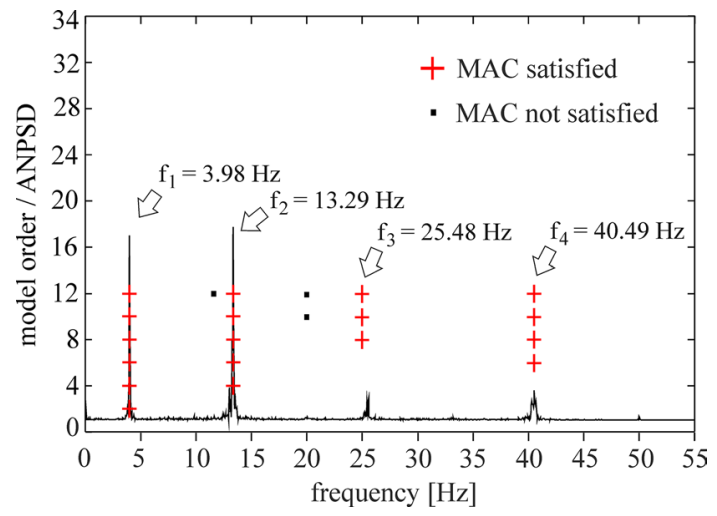

Fig. 7 Frequency stabilization diagram of the bridge (ERA) - passage of the locomotive with the speed of $10 \mathrm{~km} / \mathrm{h}$

$f_{2}=13.29 \mathrm{~Hz}, f_{3}=25.48 \mathrm{~Hz}$. The natural frequencies and their scatters indicated by PP-CA are: $f_{1}=4.26 \pm 0.35 \mathrm{~Hz}$, $f_{2}=13.49 \pm 0.35 \mathrm{~Hz}, f_{3}=25.57 \pm 0.35 \mathrm{~Hz}$. The results of ERA belong to the range of the PP-CA results.

The damping coefficient corresponding to the first natural frequency in ERA is $\xi_{1}=0.0059$; whereas, the result of logarithmic decrement method is $\xi_{1}^{\mathrm{LDD}}=0.0061$.

\section{Discussion}

The paper addresses effectiveness of two modal identification methods regarding selected irrelevant cases. The ERA method points out the irrelevant case of Vistula Mounting Fortress tower. Here, ambient vibrations are measured, free-decay character of signals is barely observable, while immediate actions are imposed on the structure. The case of KO30 bridge, where free-decay vibrations are measured, is relevant due to ERA method. While regarding the PP-CA technique, the conclusions reverse. The Vistula Mounting Fortress tower is a relevant case here; the bridge case becomes the irrelevant one. The PP-CA is a stochastic MID technique, leading to natural frequencies and mode shapes assessment, including their scatter. The ERA is a deterministic method, in which scatters of the identified modal characteristics are not considered. The study investigates the ways the considered techniques may be applied in irrelevant cases; the issues emerging here will be discussed further on.

Three mode shapes and corresponding frequencies have been identified for the bridge by means of both MID techniques, detecting convergence of results. The difference between the natural frequencies identified in ERA and PP-CA (mean value) are equal to $0.28 \mathrm{~Hz}, 0.20 \mathrm{~Hz}$ and $0.09 \mathrm{~Hz}$ subsequently. Every difference is smaller than the error of the frequencies determination in PP-CA technique (equal to $0.35 \mathrm{~Hz}$ in the considered case). Thus, the natural frequencies identified in ERA are included in the PP-CA result domain. Moreover, high correlation coefficients of modal forms estimated by both methods are obtained. Due to the first mode shape, the correlation coefficient of the ERA and PP-CA results reaches 0.9999. That proves PP-CA can be applied to short-time signals; however, statistical error of the results is increased, up to $25.8 \%$ in this case.

In the tower case, a single natural frequency and the corresponding mode shape are identified only. To check the ERA effectiveness in this irrelevant case, fifteen different time sections have been considered. In a consequence, a set of 15 ERA solutions are obtained. All of them fit in the

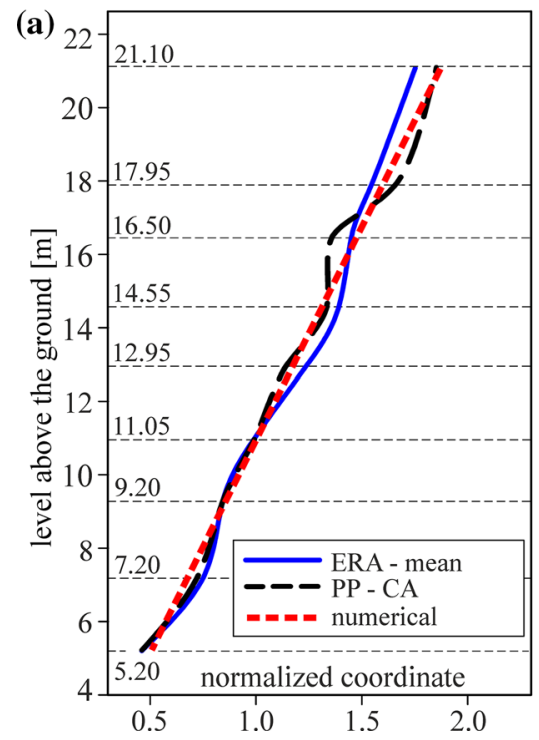

(b)
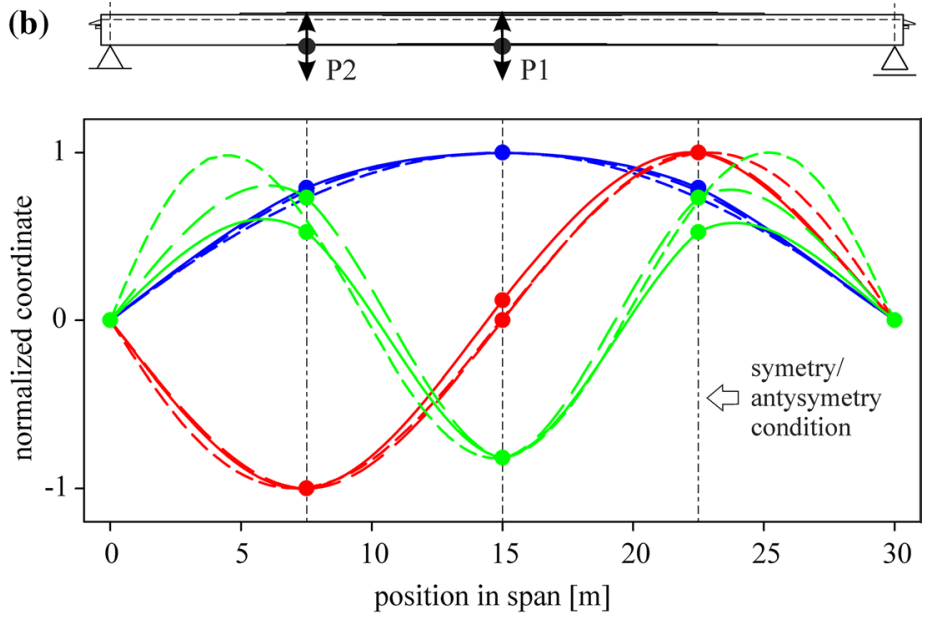

linestyles denote the following mode shape:
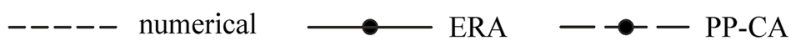

Fig. 8 Comparison between numerical and experimental mode shapes of: a tower, $\mathbf{b}$ bridge 
range of modal coordinates scatter determined in the PP-CA algorithm (five example solutions are presented in Fig. 2b). The correlation coefficients of all results calculated in relation to the mode identified in PP-CA technique are high (the range of 0.9917-0.9969). On the other hand, the modal forms obtained in ERA are visibly diverse and the true result selection is problematic. The authors propose to average the modal coordinates over the obtained in ERA set. The considered example proves that such operation approaches the mode obtained in ERA to true result (obtained in PP-CA here). This average mode shape is apparently closer to the PP-CA result than separate ERA results, see Fig. $2 b$. The correlation coefficient for the averaged ERA output and the PP-CA result is 0.9967 , close to maximum correlation coefficient of every separate pair of ERA and PP-CA outcomes. This idea of averaging corresponds to the PP-CA algorithm, in which this operation is inherent at the procedure start, where the measured signal is split into $n_{d}$ parts; next the spectral functions are computed as averages over the $n_{d}$ signal set (see Eqs. 7, 8).

The random error of mode shape coordinates determined in PP-CA is relatively high: $17.7 \%$ for the tower and $25.8 \%$ for the bridge. The scatter can be reduced by splitting the analyzed signals into a greater number of $n_{d}$ sections, but upon this action, the accuracy of natural frequencies decreases, i.e., $\Delta f$ becomes higher. An alternative solution is to take longer measurements. This issue should be balanced in each problem incorporating the PP-CA technique.

Small damping ratio is obtained for the KO30 bridge. The identified value of $\xi=0.6 \%$ is close to the bottom value recommended in EN 1991-2:2003 design standard (due to steel and complex bridges spanning above $20 \mathrm{~m}$ the recommended value is $\xi=0.5 \%$ ). The tower of Vistula Mounting Fortress is characterized by a damping ratio typical for that structural sort. In the book [23], the damping ratios identified for 19 different masonry towers are given. The values are in the range $0.014-0.078$. The most common results range $0.02-0.03$. Higher values appear in stocky towers, while the result $\xi=0.078$ corresponds to belfry with cracks. Other literature sources report similar values. In example in [24]. the authors discuss modal parameters of masonry bell tower of the Hagia Sophia church in Trabzon (Turkey). Damping ratio corresponding to the first natural frequency equals 0.0362. In the case of the bell tower of the Cathedral of Fiesole (Italy), the damping ratio is 0.0163 (see [25]). The other two towers are described in $[26,27]$. They are the bell towers of Saint Prospero and of the Santa Maria Maggiore cathedral (Italy), respectively. The identified damping coefficients corresponding to the first natural frequencies are equal to 0.0198 and 0.016 .

The modal forms of the tower identified by ERA discover that all fifteen results are similar in an approximate height range of $0-11 \mathrm{~m}$ above the ground level (see Fig. 2b). In higher structural sections, the variants split to different coordinates. Such phenomenon is not observed in the bridge. As proved in [28], the cantilever-like tower structure shows increased modal displacements above the damaged section. Thus, the observed distortions in the modal shape indicate a local damage just below the section of the distortion onset. Such hypothesis corresponds to the results of the paper [13], the case of damage detected for this tower 9-m high above the ground. This result is confirmed by visual inspectionvertical cracks approximately $2-\mathrm{m}$ long are visible around the tower at this height. The paper [13] addresses numerical model of the tower. The model has been created in a commercial SOFiSTiK software, employing Finite Element Method. The model reflects 3-dimensional structural geometry. The material properties and boundary conditions have been determined by means of the model validation to the first three natural frequencies and modal forms identified experimentally. The numerical mode shape corresponding to the one discussed in the paper is presented in Fig. 8a with the average ERA result and the mode identified by the PP-CA technique. Similarity of both experimental results and the numerical mode is observable. However, the mode shape curvature at an approximate level $16.50 \mathrm{~m}$ indicated by the PP-CA technique suggests local damage presence. The authors could not see any defect in that region; nevertheless, further inspection is necessary there. To adjust the numerical mode shape to the experimental one, the model is directed to further validation. In future research, elastic modulus of the brick masonry is bound to be reduced in the cracked sections. Such method of crack consideration in the numerical model is discussed, e.g., in the paper [29].

The three-dimensional numerical model of the bridge has been developed with the use of the same software and validated on the basis of first two identified bending modes, as presented in [30]. Numerical and experimental modes of the bridge are compared in Fig. 8b. Planar sections of the first three bending modes are presented, related to the vertical acceleration measurements. The experimentally determined modes are visualized by means of spline functions to provide interpolation between the measured coordinates, marked by dots. Close similarity of numerical and experimental mode shapes is observed. The third mode comparison detects some visible discrepancy in the bridge span edges. Apparently additional sensor is intended to be located at the distance of $4.8 \mathrm{~m}$ from the supports to identify extreme modal displacements.

\section{Conclusions}

The discussed results prove that PP-CA can be successfully applied to short-time deterministic signals. However, statistical errors of modal identification results are 
relatively high then. On the other hand, the ERA method is applicable to ambient vibrations supplemented by immediate excitations. Nevertheless, while ambient vibrations are stochastic processes, the subsequent, identified modal results should be considered as random variables. The ERA result is deterministic, so the random data nature should be captured by a population of dozen signal intervals analysis and the modal results should be averaged over the obtained set. Such methodology approaches the modes identified in the ERA to the forms identified in the PP-CA, dedicated to ambient vibration analysis. An asset of two different MID methods application to a given case is a possibility of the results confirmation. The advantage is taken especially in the case of signals considered deterministic, e.g., free decay, suitable for the ERA-the results of PP-CA strongly resemble the ERA results in the considered example.

In the damaged tower region, the mode shapes split up to different coordinates due to all ERA realizations. This observation can initiate a novel damage index development.

Acknowledgements The author expresses a gratitude to Authorities of Museum of Gdańsk City, Department of Vistula Mounting Fortress, for sharing photos of the Fortress.

\section{Compliance with ethical standards}

Conflict of interest The authors declare that they have no conflict of interest.

Open Access This article is licensed under a Creative Commons Attribution 4.0 International License, which permits use, sharing, adaptation, distribution and reproduction in any medium or format, as long as you give appropriate credit to the original author(s) and the source, provide a link to the Creative Commons licence, and indicate if changes were made. The images or other third party material in this article are included in the article's Creative Commons licence, unless indicated otherwise in a credit line to the material. If material is not included in the article's Creative Commons licence and your intended use is not permitted by statutory regulation or exceeds the permitted use, you will need to obtain permission directly from the copyright holder. To view a copy of this licence, visit http://creativecommons.org/licenses/by/4.0/.

\section{References}

1. Maia NMM, Silva JMM. Theoretical and experimental modal analysis. Baldock: Research Studies Press Ltd.; 1997.

2. Zhang L. An overview of major developments and issues in modal identification. In: Proceedings of 22nd international modal analysis conference (IMAC), Detroit; 2004.

3. Bendat J, Piersol A. Engineering applications of correlation and spectral analysis. New York: Wiley; 1980.

4. Zwolski J, Bień J. Modal analysis of bridge structures by means of forced vibration tests. J Civ Eng Manag. 2011;17(4):590-9.

5. Gazzani V, Poiani M, Clementi F, Milani G, Lenci S. Modal parameters identification with environmental tests and advanced numerical analyses for masonry bell towers: a meaningful case study. Procedia Struct Integr. 2018;11:306-13.
6. Brownjohn JMW. Ambient vibration studies for system identification of tall buildings. Earthq Eng Struct D. 2003;25:1-25.

7. Chen G, Omenzetter P, Beskhyroun S. Operational modal analysis of an eleven-span concrete bridge subjected to weak ambient excitations. Eng Struct. 2017;151:839-60.

8. Bień J, Kużawa M, Kamiński T. Validation of numerical models of concrete box bridges based on load test results. Arch Civ Mech Eng. 2015; 15:1046-60.

9. Nayeri RD, Tasbihgoo F, Wahbeh M, Caffrey JP, Masri SF, Conte JP, Elgamal A. Study of time-domain techniques for modal parameter identification of a long suspension bridge with dense sensor arrays. J Eng Mech. 2009;135:669-83.

10. Szafrański M. Dynamics of the small-span railway bridge under moving loads. In: MATEC Web conference 2019, vol. 262, no 10014, pp 1-8.

11. Poprawa G, Salamak M, Pradelok S, Łaziński P. Operational modal analysis in model updating of a truss railway bridge. In: Proceedings of 7 th international operational modal analysis conference (IOMAC), Ingolstadt; 2017. pp. 9-18.

12. Stutz LT, Rangel ICSS, Rangel LS, Corrêa RAP, Knupp DC. Structural damage identification built on a response surface model and the flexibility matrix. J Sound Vib. 2018;434:284-97.

13. Tomaszewska A. Influence of statistical errors on damage detection based on structural flexibility and mode shape curvature. Comput Struct. 2010;88:154-64.

14. Juang JN, Pappa RS. An eigensystem realization algorithm for modal parameter identification and model reduction. J Guid Control Dyn. 1985;8:620-7.

15. Hollkamp JJ, Gordon RW. Modal test experiences with a jet engine fan model. J Sound Vib. 2001;248:151-65.

16. Rusinski E, Dragan S, Moczko P, Pietrusiak D. Implementation of experimental method of determining modal characteristics of surface mining machinery in the modernization of the excavating unit. Arch Civ Mech Eng. 2012;12:471-6.

17. Bernagozzi G, Mukhopadhyay S, Betti R, Landi L, Diotallevi PP. Output-only damage detection in buildings using proportional modal flexibility-based deflections in unknown mass scenarios. Eng Struct. 2018;167:549-66.

18. Alvin KF, Robertson AN, Reich GW, Park KC. Structural system identification: from reality to models. Comput Struct. 2003;81:1149-76.

19. Juang JN. Applied system identification. New Jersey: Prentice-Hall PTR; 1994.

20. Bendat J. Statistical errors in measurement of coherence functions and input/output quantities. J Sound Vib. 1978;59:405-21.

21. Taylor JR. An Introduction to error analysis: the study of uncertainties in physical measurements. 2nd ed. Sausalito: University Science Books; 1997.

22. Tomaszewska A, Szymczak C. Identification of the Vistula Mounting tower model using measured modal data. Eng Struct. 2012;42:342-8.

23. Bull JW. Computational modelling of masonry, brickwork and blockwork structures. Stirling: Saxe-Coburg Publications; 2001.

24. Bayraktar A, Türker T, Sevim B, Altunisik AC, Yildirim F. Modal parameter identification of Hagia Sophia bell-tower via ambient vibration test. J Nondestruct Eval. 2009;28:37-47.

25. Bru D, Ivorra S, Betti M, Adam JM, Bartoli G. Parametric dynamic interaction assessment between bells and supporting slender masonry tower. Mech Syst Signal Process. 2019;129:235-49.

26. Vincenzi L, Bassoli E, Ponsi F, Castagnetti C, Mancini F. Dynamic monitoring and evaluation of bell ringing effects for the structural assessment of a masonry bell tower. J Civ Struct Health Monit. 2019;9:439-58.

27. Zanotti Fragonara L, Boscato G, Ceravolo R, Russo S, Ientile S, Pecorelli ML, Quattrone A. Dynamic investigation on the 
Mirandola bell tower in post-earthquake scenarios. Bull Earthq Eng. 2017;15:313-37.

28. Pandey AK, Biswas M, Samman MM. Damage detection from changes in curvature mode shapes. J Sound Vib. 1991;145:321-32.

29. Clementi F, Pierdicca A, Formisano A, Catinari F, Lenci S. Numerical model upgrading of a historical masonry building damaged during the 2016 Italian earthquakes: the case study of the Podesta palace in Montelupone (Italy). J Civ Struct Health Monit. 2017;7:703-17.
30. Szafrański M. Dynamic analysis of the railway bridge span under moving loads. Roads and Bridges (Drogi i Mosty). 2018;17:299-316.

Publisher's Note Springer Nature remains neutral with regard to jurisdictional claims in published maps and institutional affiliations. 